GU J Sci, Part C, 6(4): 729-740 (2018)

Gazi Üniversitesi
Fen Bilimleri Dergisi
PART C: TASARIM VE TEKNOLOJI
http://dergipark.gov.tr/gujsc

\title{
Polianilin/Aljinat Malzemesinin Asit Violet 90 Boyar Maddesinin Gideriminde Kullanılması: Kinetik Ve İzoterm Değerlendirmesi
}

\author{
Müjgan Okur ${ }^{1, *}$, Filiz Aktı², Ayşenur Çetintaş ${ }^{1}$ \\ ${ }^{I}$ Gazi Üniversitesi, Mühendislik Fakültesi, Kimya Mühendisliği Bölümü, 06570, ANKARA \\ ${ }^{2}$ Hitit Üniversitesi, Mühendislik Fakültesi, Kimya Mühendisliği Bölümü, 19030, ÇORUM
}

\begin{abstract}
$\ddot{O ̈ z}$
$\underline{\text { Makale Bilgisi }}$

Başvuru: 25/01/2018

Düzeltme: 25/07/2018

Kabul: 07/08/2018

\section{Anahtar Kelimeler}

Polianilin

Bu çalışmada Polianilin/Aljinat (PANİ/SA) adsorbent malzemesi Asit Violet 90 (AV 90) boyar maddesinin adsorpsiyonunda kullanılmıştır. PANİSA'nın karakterizasyonu Fourier Dönüşüm Kızılötesi Spektroskopisi (FTIR) ile analiz edilmiştir. pH (2-6), PANI/SA küre sayısı (10-50 adet) ve AV 90 derişiminin $(25-200 \mathrm{mg} / \mathrm{L})$ boyar madde giderimine etkisi incelenmiştir. En yüksek boyar madde giderimi $\mathrm{pH}$ 2'de elde edilmiştir. AV 90 adsorpsiyon izotermlerini belirlemek için Langmuir, Freundlich ve Temkin modelleri kullanılmıştır. Boyar madde derişimi 25-200 mg/L aralığında iken Langmuir modelinden elde edilen maksimum adsorpsiyon kapasitesi $\left(\mathrm{q}_{\mathrm{m}}\right)$ değeri $138,9 \mathrm{mg} / \mathrm{g}$ olarak bulunmuş̧tur. Adsorpsiyon kinetiğinin belirlenmesi için Yalancı birinci derece ve ikinci derece kinetik modelleri kullanılmış ve Yalancı ikinci derece kinetik modelin deney verileri ile daha uyumlu olduğu görülmüştür. Deney sonuçları PANI/SA adsorbentinin AV 90 boyar maddesinin adsorpsiyonu için oldukça iyi bir alternatif olduğunu göstermiştir.
\end{abstract}

Aljinat

Asit Violet 90

Adsorpsiyon

Adsorpsiyon kinetiği

\section{Keywords}

Polyaniline

Alginaet

Acid Violet 90

Adsorption

Adsorption kinetics

\section{Use of Polyaniline/Alginate Composite Material in the Adsorption of Acid Violet 90 Dye:Kinetics and Isotherm Evaluation}

\begin{abstract}
In this study, Polyaniline/Alginate (PANI/SA) material was used to adsorption of Acid Violet 90 (AV 90) dye. The characterization of PANI/SA was analyzed by Fourier transform infrared spectroscopy (FTIR). The effect of $\mathrm{pH}$ (2-6), number of PANI/SA beads (10-50 adet) and concentration of AV $90(25-200 \mathrm{mg} / \mathrm{L})$ on removal of dye were examined. The highest dye removal was obtained at $\mathrm{pH}$ 2. Langmuir, Freundlich and Temkin models were used to determine AV 90 adsorption isotherms. The maximum adsorption capacity $\left(\mathrm{qm}_{\mathrm{m}}\right)$ value obtained from the Langmuir model in the range of $25-200 \mathrm{mg} / \mathrm{L}$ dye concentrations was found to be $138.9 \mathrm{mg} / \mathrm{g}$. To determine the adsorption kinetics, Pseudo first order and second order kinetic models were used and it was found that the Pseudo second order kinetic model was more compatible with the experimental data. Experimental results have shown that PANI/SA adsorbent is a very good alternative for adsorption of AV 90 dye.
\end{abstract}

\section{GÍRIŞ (INTRODUCTION)}

Sentetik boyar maddeler, tekstil, kozmetik, kağıt, plastik ve deri gibi bir çok endüstriyel alanda kullanılmakta ve bu sektörlerin atık suları önemli miktarlarda boyar madde içermektedir [1-3]. Günümüzde dünya çapında yaklaşık 100000 ‘in üzerinde boya ve pigment türü kullanılmakta ve yılda 700000 tonun üzerinde boya üretilmektedir. Üretilen bu boyar maddelerin yaklaş1k \% 20-30'u tekstil boyama proseslerinde etkin boyamanın yapılamaması sonucunda atık sulara karışmaktadır [4]. Toksik etkiye sahip olan ve sucul ortamda parçalanmaya dirençli olan bu organik boyar maddeler sucul yaşamı ve insan hayatını tehlikeye atmaktadır. Sucul ortamlara karışan bu kirleticiler, suyun 1 şık geçirgenliğini düşürerek fotosentetik aktiviteyi etkileyebilmektedirler [5,6]. Ayrıca boyarmaddelerin bazı sucul organizmalarda birikmesi toksik ve kanserojenik ürünlerin meydana gelme riskini de beraberinde getirmektedir. $\mathrm{Bu}$ 
bağlamda boyarmadde içeren tekstil endüstrisi atık sularının renk giderim prosesleri çevresel açıdan önem kazanmaktadır [7]. Bunların yanı sıra, tekstil endüstrisinde kullanılan boyarmaddelerin kompleks aromatik moleküler yapıları, bu boyarmaddelerin oldukça kararlı ve parçalanmaya karşı dirençli hale gelmesine neden olmaktadır [8]. Bu nedenle, tekstil atık sularının karakterizasyonu, boyaların kimyasal yapısındaki farklı1ıklardan ve boyama prosesinin değişim göstermesinden dolayı oldukça zordur [7]. Boyar maddeleri atık sulardan gidermek için fiziko-kimyasal yöntemler (adsorpsiyon, membran prosesleri, koagulasyon/flokulasyon, elektrokoagulasyon vb.) ve biyolojik yöntemler (anaerobik, aerobik) uygulanmaktadır [9]. Adsorpsiyon boyarmadde gideriminde kullanılan verimli, kolay uygulanabilen, ekonomik ve en çok tercih edilen metotlardan birisidir [4,10,11]. Adsorpsiyon proseslerinde genellikle düşük maliyetli ve kolay bulunabilen adsorbentler ile etkili boyar madde işlemleri gerçekleştirilebilmektedir [9].

Polianilin (PANI) içerdiği amin ve imin gruplarından dolayı, organik ve inorganik kirleticilerin atık sulardan gideriminde tercih edilen iletken bir polimerdir [11]. Ayrıca PANİ kolay sentezlenebilen, yapısal olarak kararlı ve düşük maliyetli bir monomerdir [13,14]. Aljinat düz zincir yapıda, $1 \rightarrow 4$ bağlı $\beta$-D mannuronik asit ve $\alpha$-L guluronik asit birimlerinden oluşan bir kopolimerdir $[15,16]$. Aljinat negatif yüklü olmasından dolayı ağır metallerin ve katyonik boyar maddelerin gideriminde kullanılabilecek uygun bir polimerdir [17]. Yapılan çalışmalar aljinatın $\mathrm{Fe}_{3} \mathrm{O}_{4}$ [2], montmorillonit [5], grafen oksit [17] gibi malzemelerle nanokompozitleri şeklinde kullanılmasının hem aljinatın mekanik özelliklerinin hem de adsorpsiyon özelliklerinin geliştirilmesine katkıda bulunduğunu göstermişlerdir. Aljinat ve PANI'nin oluşturduğu malzeme ise $\mathrm{Cu}(\mathrm{II}), \mathrm{Pb}(\mathrm{II}), \mathrm{Cr}(\mathrm{VI})$ adsorpsiyonunda kullanılmıştır $[15,18]$. Bu çalışmada ise Aljinat ve PANI adsorbenti (PANI/SA) Asit Violet 90 (AV90) boyar maddesinin adsorpsiyonunda kullanılacaktır. PANI/SA ile AV 90 adsorpsiyonuna $\mathrm{pH}$, adsorbent miktarı ve AV 90 derişimi parametrelerinin etkisi incelenecektir. Adsorpsiyon izotermlerinin belirlenmesi için Langmuir, Freundlich ve Temkin adsorpsiyon izoterm modellerinden yararlanılmıştır. Adsorpsiyon kinetiğinin belirlenmesi için Yalancı birinci derece (Pseudo-first order) ve Yalancı ikinci derece (Pseudo-second order) kinetik modeller kullanılmıştır.

\section{DENEYSEL YÖNTEM (EXPERIMENTAL METHOD)}

\subsection{Adsorbentin Hazırlanması (Preparation of Adsorbent)}

Polianilin/Aljinat (PANİ/SA) kürelerin hazırlanması için öncelikle sodyum aljinat $100 \mathrm{~mL}(\% 2 \mathrm{w} / \mathrm{v})$ saf suda çözüldü. Homojen bir karışım elde etmek için yaklaşık 2 saat manyetik karıştırıcıda karıştırıldı. $2 \mathrm{~mL}$ anilin $100 \mathrm{~mL} 0,1 \mathrm{M} \mathrm{HCl}$ içerisinde çözüldü ve sodyum aljinat, anilin içerisine enjektör ile damla damla eklenerek kürelerin oluşması sağlandı ve oluşan küreler yaklaşık 3 saat manyetik karıştırıcıda karıştırıldı. Karışımın içerisine oda sıcaklığında polianilin polimerizasyonu için başlatıcı olarak potasyum persülfat $\left(\mathrm{K}_{2} \mathrm{~S}_{2} \mathrm{O}_{8}\right)(5,9 \mathrm{~g} / 100 \mathrm{~mL} 1 \mathrm{M} \mathrm{HCl})$ yavaşça eklendi. Karışım yaklaşık 2 saat manyetik karıştırıcıda karıştırıldıktan sonra çözelti süzüldü ve saf su ile yıkandı. Hazırlanan PANI/SA malzemesi buzdolabında $+4{ }^{\circ} \mathrm{C}$ saklandi.

\subsection{Adsorpsiyon Deneyleri (Adsorption Experiments)}

Deneyler $100 \mathrm{~mL}$ çalışma hacmine sahip $250 \mathrm{~mL}$ 'lik erlenlerde, çalkalamalı su banyosunda $25^{\circ} \mathrm{C}$ sıcaklıkta ve $100 \mathrm{rpm}$ karıştırma hızında gerçekleştirilmiştir. Deneylerde incelenen parametreler aşağıda listelenmiştir. Deneylerde boyarmadde adsorpsiyonuna $\mathrm{pH}(2,3,4$ ve 6), küre sayısı $(10,20,30,40$ ve 50 adet ) ve boyarmadde derişiminin $(25,50,100,150$ ve $200 \mathrm{mg} / \mathrm{L})$ etkisi incelenmiştir. 10 adet kürenin kuru ağırlığ 1,2 mg olarak belirlenmiştir. Çözeltilerin pH’1 $1 \mathrm{M} \mathrm{HCl}$ ve $1 \mathrm{M} \mathrm{NaOH}$ kullanılarak ayarlanmıştır. Deneyler esnasında belirli zaman aralıklarında alınan numunelerdeki boyarmadde derişimi UVSpektrofotometresi ile $523 \mathrm{~nm}$ dalga boyunda belirlenmiştir. Adsorbent yüzeyindeki fonksiyonel grupları belirlemek için Bruker Vertex 70/70v marka FTIR (Fourier transforms infrared) cihazı ile 400-4000 $\mathrm{cm}^{-1}$ dalga boyu aralığında spektrum taraması yapılmıştır. Adsorpsiyon sonrası \% boyar madde giderimi ve boyar madde giderim kapasitesi değerleri aşağıdaki eşitliklerden yararlanılarak hesaplanmıştır.

$$
\% \text { Boyar madde Giderimi }=\frac{\mathrm{C}_{o}-\mathrm{C}_{e}}{\mathrm{C}_{o}} 100
$$




$$
\text { Boyar madde Giderim Kapasitesi }=\frac{\mathrm{C}_{o}-\mathrm{C}_{e}}{\mathrm{~m}} V
$$

$\mathrm{Bu}$ eşitliklerde $\mathrm{C}_{\mathrm{o}}$ ve $\mathrm{C}_{\mathrm{e}}$ ortamda başlangıçtaki ve dengedeki boyar madde derişimi (mg/L), $\mathrm{m}$ kürenin kuru kütlesi (g), V çözelti hacmidir (L).

\section{BULGULAR ve TARTIŞMA (RESULTS and DISCUSSION)}

\subsection{FTIR Analizi (FTIR Analysis)}

Şekil 1 SA, PANI ve PANI/SA 'ya ait FTIR spektrumlarını göstermektedir. SA'ya ait piklerde $3313 \mathrm{~cm}^{-1}$ $\mathrm{O}-\mathrm{H}$ gerilmelerini, 1595 ve $1417 \mathrm{~cm}^{-1}$ ' $\mathrm{de}-\mathrm{COO}^{-}$nedeniyle olan asimetrik ve simetrik gerilmeler, $1024 \mathrm{~cm}^{-}$ ${ }^{1}$ 'de-C-O-C- gerilmeleri görülmektedir [1,5]. SA'ya ait olan $900-1200 \mathrm{~cm}^{-1}$ 'deki pikler ise eter gruplarının -O-C-O- gerilmelerini ve alkol gruplarının -C-O- gerilmelerini göstermektdir [16]. PANI'ye ait spektrumlarda ise $1560 \mathrm{~cm}^{-1}$ and $1460 \mathrm{~cm}^{-1}$ 'de görülen pikler, PANI'’nin karakteristik quinoid ve benzenoid halkalarına ait $\mathrm{C}=\mathrm{C}$ gerilme titreşimlerini göstermektedir. Yine PANI'ye ait 1284 ve $1232 \mathrm{~cm}^{-1}$ 'deki pikler ise C-N'e karşılık gelmektedir [16]. PANİ/SA'ya ait FTIR spektrumlarında 1560, 1460, 1232 [18], 871 ve $783 \mathrm{~cm}^{-1}$ 'e karşılık gelen pikler PANI'ye ait piklerdir. PANİ/SA'da $1712 \mathrm{~cm}^{-1}$ 'de ortaya çıkan yeni pik ise SA'daki - $\mathrm{COO}^{-6}$ yu göstermektedir. Bu pik, PANİ/SA eldesi sirasında SA'daki - $\mathrm{COO}^{-}$grupları ile PANI'deki protonlanmış amin grupları arasındaki elektrostatik etkileşim sebebiyle olabileceği düşünülmektedir $[15,18]$.

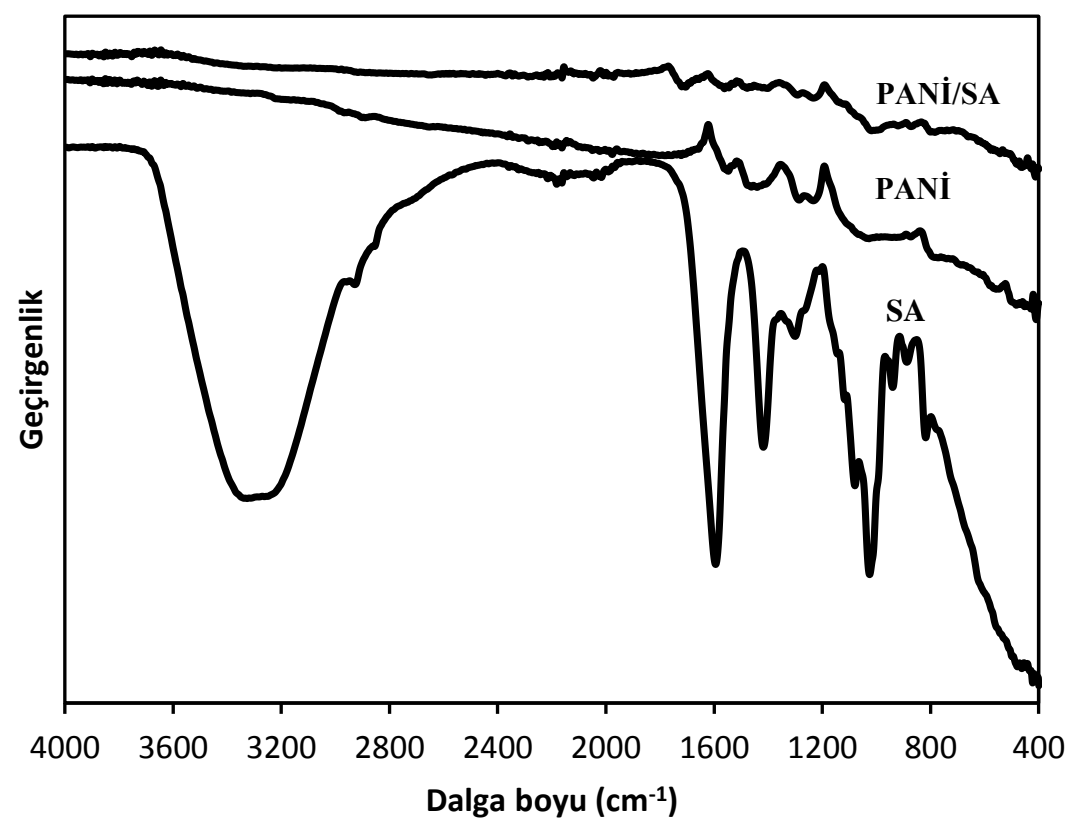

Şekil 1. SA, PANI ve PANI/SA 'ya ait FTIR spektrumlart

\section{2. pH'ın Boyar Madde Adsorpsiyonu Üzerine Etkisi (The effect of pH on Dye Adsorption)}

Organik boyarmaddelerin adsorpsiyonunda oldukça önemli parametrelerden birisi ortam pH'ıdır. Çünkü $\mathrm{pH}$ adsorbentin yüzey yükünü ve aktif bölgelerini etkiler [19]. Boyar madde adsorpsiyonuna $\mathrm{pH}$ etkisini belirlemek için $\mathrm{pH}$ 2,3,4,6 olarak değiştirilmiştir. pH etkisinin incelendiği deneylerde $50 \mathrm{mg} / \mathrm{L}$ boyar madde derişiminde ve 10 adet PANİ/Aljinat küresi kullanılmış ve adsorpsiyon deneyleri 72 saat boyunca takip edilmiştir. Adsorpsiyon deneylerinin 48. saatte dengeye geldiği görülmüştür. Farklı başlangıç pH değerlerinde yüzde boyar madde gideriminin zamanla değişimi Şekil 2'de verilmiştir. En yüksek boyar madde giderimi pH 2'de \% 50,43 olarak elde edilmiştir (Şekil 3). Bu pH'ta elde edilen boyar madde giderim kapasitesi 139,2 mg/g'dır. pH 2'den 3'e arttırıldığında boyarmadde gideriminde hızlı bir düşüş olmuştur. $\mathrm{pH}$ 3'ün üzerine çıkarıldığında giderim yüzdesi daha yavaş bir azalma eğilimi göstermiştir. AV 90 boyarmaddesi anyonik bir boyar maddedir. Düşük $\mathrm{pH}$ değerlerinde PANI/SA yüzeyinde pozitif yüklü 
bölgelerin sayısı artabilmekte ve bu durumda negatif yüklü AV 90 ile pozitif yüklü PANİ/SA yüzeyi arasındaki elektrostatik çekim kuvvetlerinin artmasından dolayı boyar madde giderim yüzdesi de artmaktadır. Benzer sonuç Kongo kırmızısı boyar maddesinin $\mathrm{PANI} / \mathrm{Bi}_{2} \mathrm{WO}_{6}$ adsorbenti ile gideriminde [3], kitosan-PANİ/ZnO kompozit malzemesi ile reaktif orange 16 adsorpsiyonunda [10], aljinatmontmorillonit-PANİ kompozit malzemesi ile reaktif orange 16 adsorpsiyonunda [16] elde edilmiştir.

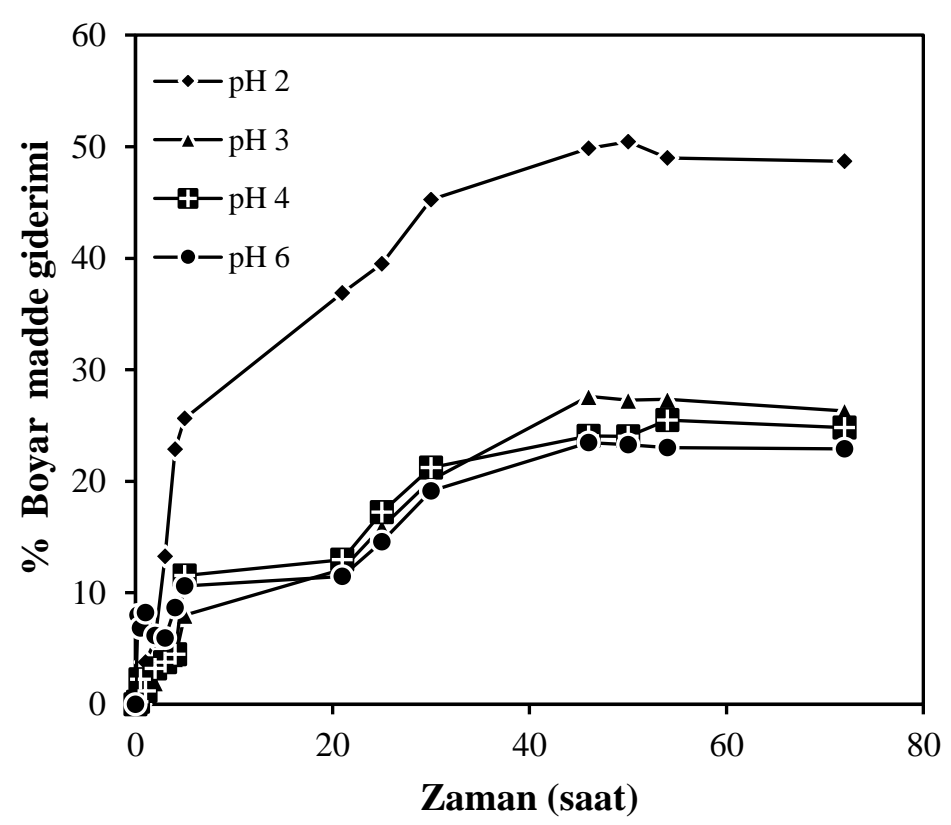

Şekil 2. Farkl $p H$ 'larda \% boyarmadde gideriminin zamanla değişimi $(50 \mathrm{mg} / \mathrm{L}$ boyarmadde, 10 adet küre)

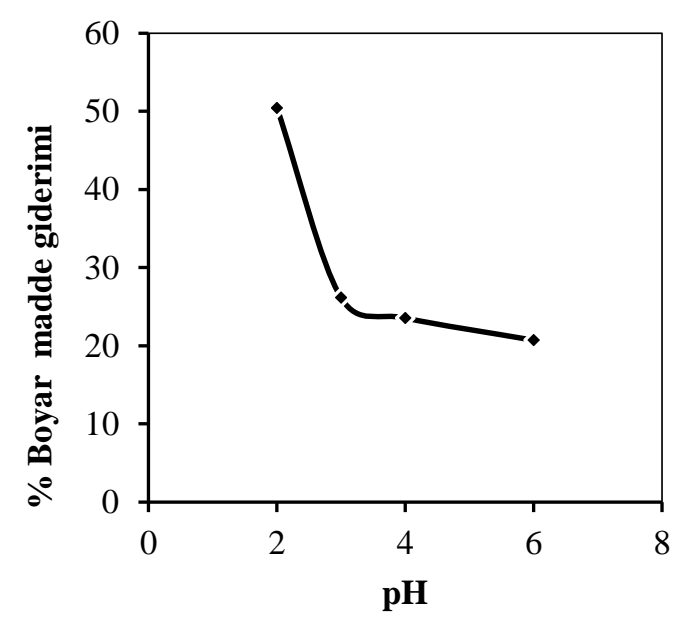

Şekil 3. $p H$ 'ın \% boyarmadde giderimine etkisi (50 mg/L boyarmadde, 10 adet küre)

\subsection{Küre Sayısının Etkisi (Effect of Bead Number)}

Boyar madde adsorpsiyonuna küre sayısının etkisi $\mathrm{pH}$ 2'de ve $50 \mathrm{mg} / \mathrm{L}$ boyarmadde derişiminde incelenmiştir. Küre sayısı 10, 20, 30, 40 ve 50 adet olarak seçilmiştir. Küre sayısının boyar madde giderimine etkisi Şekil 4'te ve giderim kapasitesine etkisi Şekil 5 'te verilmiştir. Küre sayısı miktarı arttıkça boyarmadde giderim yüzdesinin arttığı gözlemlenmiştir. Küre sayısı 10'dan 50'ye arttırıldığında boyar madde giderim yüzdesi \% 54,3'ten \% 94,4'e artmıştır. Az miktarda kürenin bulunduğu numunelerde düşük giderim, çok miktarda kürenin bulunduğu numunelerde yüksek giderim gözlenmiştir. $\mathrm{Bu}$ durum küre miktarı arttıkça boyar madde adsorpsiyonu yapabilecek aktif bölgelerin artmasından dolayıdır. Benzer 
sonuç Aljinat/PANİ ile Cr(VI) adsorpsiyonunda [18] gözlenmiştir. Diğer yandan küre sayısı 10'dan 50'ye arttırıldığında boyar madde giderim kapasitesi $171 \mathrm{mg} / \mathrm{g}$ 'dan 59,26 mg/g'a değişmiştir (Şekil 5). Bu çalışmada optimum küre sayısı 50 adet olarak seçilmiştir.

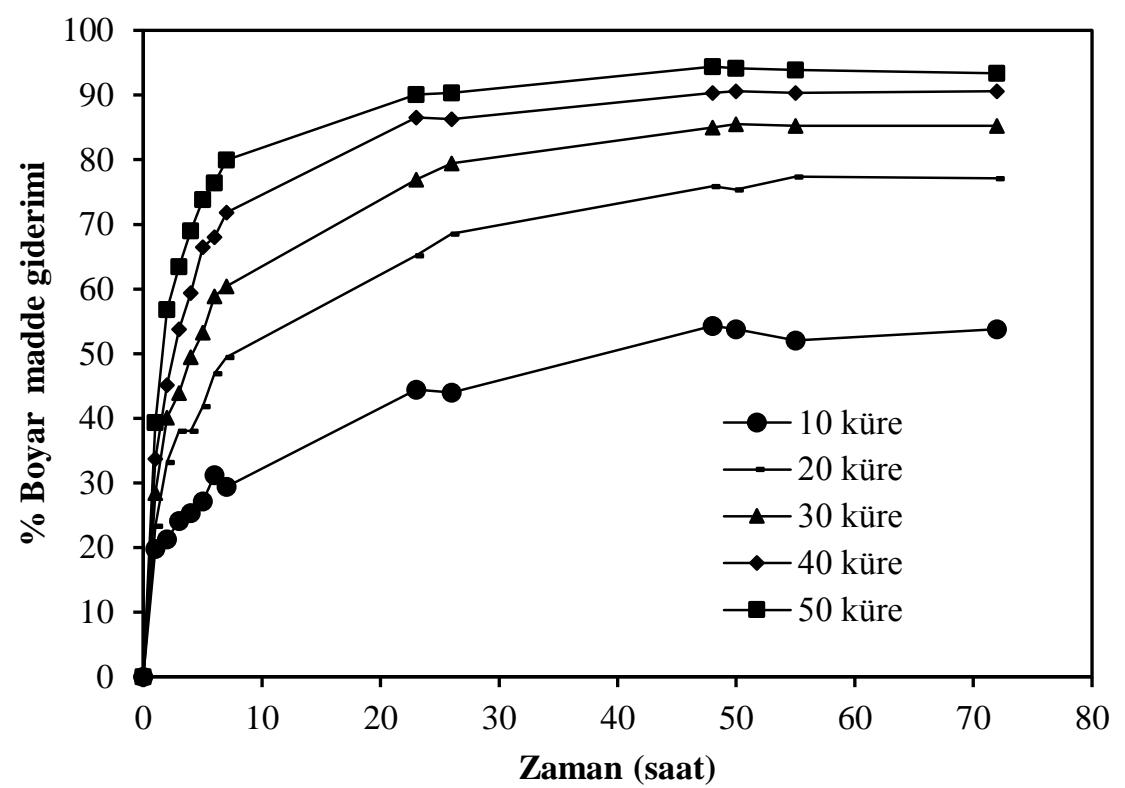

Şekil 4. Farklı küre sayılarında zamanla \% boyar madde giderimi ( $\mathrm{pH}=2,50 \mathrm{mg} / \mathrm{L}$ boyar madde)

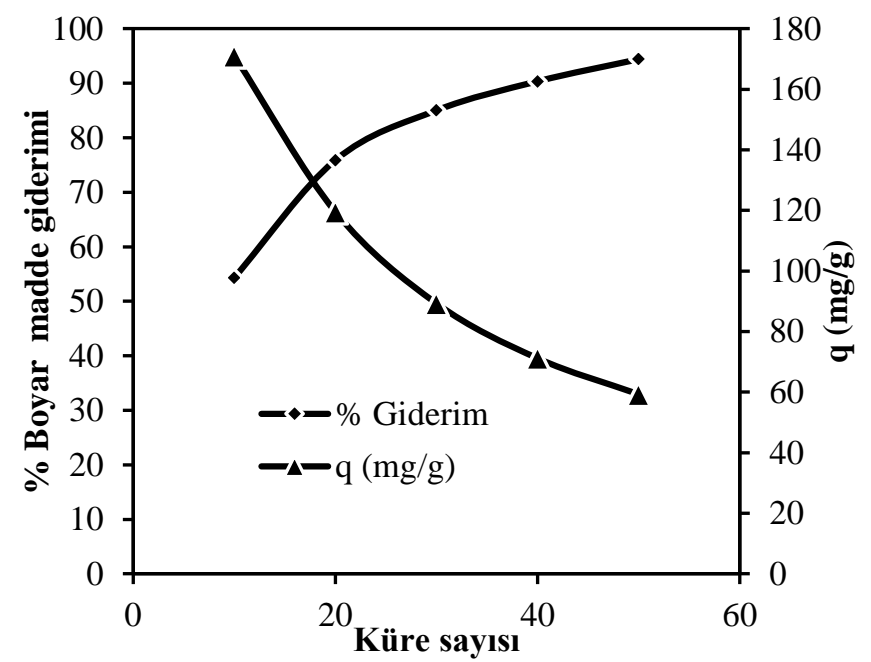

Şekil 5. Küre sayısının \% boyar madde giderimine ve giderim kapasitesine etkisi ( $\mathrm{pH}=2,50 \mathrm{mg} / \mathrm{L}$ boyar

madde)

\subsection{Boyar madde Derişiminin Etkisi (Effect of Dye Concentration)}

Boyar madde derişiminin 25-200 mg/L aralığında değiştirildiği deneylerde 50 adet küre, kullanılmıştır. Başlangıç boyar madde derişimi $25 \mathrm{mg} / \mathrm{L}$ 'den $200 \mathrm{mg} / \mathrm{L}$ 'ye arttıkça \% boyar madde giderimi \% 100'den \% 63'e değişmiştir (Şekil 6). Diğer yandan boyar madde derişimi arttıkça boyar madde giderim kapasitesi (q, mg/g) 26 mg/g'dan 134,3 mg/g'a artmıştır (Şekil 7). Boyar madde derişiminin artmasıyla PANí/SA sorbenti ile AV 90 arasındaki kütle transferi direncinin üzerinde gerekli olan itici gücü sağlamakta ve böylece boyar madde adsorpsiyonu artmaktadır. Literatürde yapılan çalışmalarda farklı boyar madde derişimleri için farklı boyar madde giderim kapasiteleri elde edilmiştir. Çapraz bağlı aljinat küreler ile 200 $\mu \mathrm{mol} / \mathrm{L}$ Metilen Mavisi adsorpsiyonunda 572,7 mg/g giderim kapasitesi (Lu ve diğ., 2015), 61,2 mg/L Brilliant Green boyarmaddesinin adsorpsiyonunda PANİ/Gümüş ile 20,92 mg/g giderim kapasitesi [12], 
$200 \mathrm{mg} / \mathrm{L}$ Congo Red boyar maddesinin PANi/Bi $2 \mathrm{WO}_{6}$ sorbenti ile adsorpsiyonunda 142,92 mg/g giderim kapasitesi [3] elde edilmiştir.

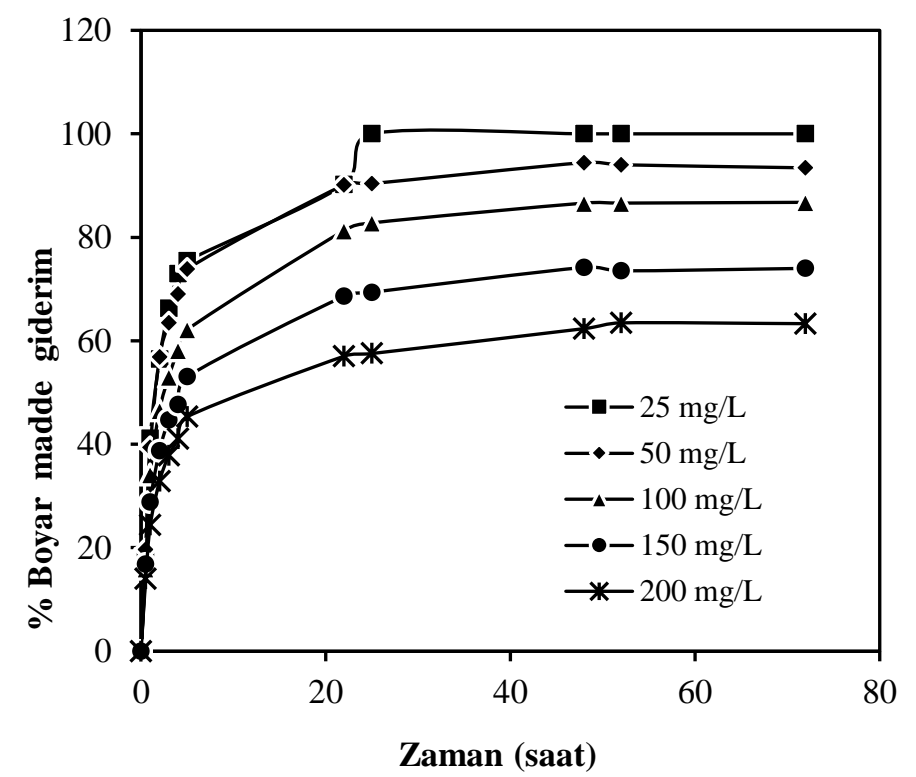

Şekil 6. Boyarmadde derişiminin boyar madde giderimine etkisi ( $p H=2,50$ küre)

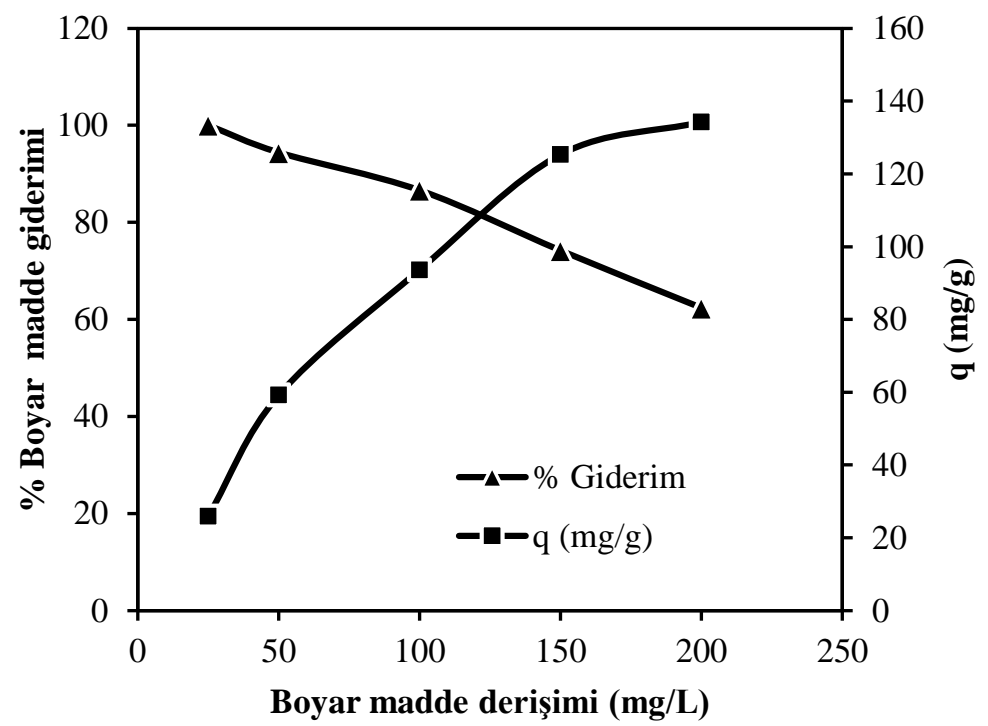

Şekil 7. Boyarmadde derişiminin \% boyar madde giderimine ve giderim kapasitesine etkisi $(p H=2,50$ küre)

\subsection{Adsorpsiyon İzotermleri (Adsorption Isoterms)}

Aljinat/polianilin kürelerinin AV 90 gideriminde sorbent olarak kullanıldığı deneylerde Langmuir, Freundlich ve Temkin adsorpsiyon izoterm modelleri kullanılmıştır. Bu izotermler kullanılırken boyar madde derişimi 25-200 mg/L olarak değiştirilmiştir. Langmuir adsorpsiyon izoterm modeli adsorpsiyon preseslerini incelemede yaygın olarak kullanılmaktadır. Bu model adsorpsiyonun tek tabakalı gerçekleştiğini ve her bir adsorplayıcı bölgenin aynı adsorpsiyon gücüne sahip olduğunu kabul etmektedir [20]. Langmuir eşitliğinin doğrusal formu Eş. 3'de verilmiştir.

$$
\frac{C_{e}}{q_{e}}=\frac{1}{K_{L_{L}} q_{m}}+\frac{C_{e}}{q_{m}}+
$$


$\mathrm{C}_{\mathrm{e}}$ : Boyar maddenin denge derişimini $(\mathrm{mg} / \mathrm{L}), \mathrm{q}_{\mathrm{e}}$ : dengede gram adsorbent başına adsorplanan boyar madde miktarın $(\mathrm{mg} / \mathrm{g})$, $\mathrm{q}_{\mathrm{m}}$ : Maksimum adsorpsiyon kapasitesini (mg/g) ve $\mathrm{K}_{\mathrm{L}}$ : Langmuir sabitini $(\mathrm{L} / \mathrm{mg})$ ifade etmektedir. Bu eşitliğin $\mathrm{C}_{\mathrm{e}}$ 'ye karşı $\mathrm{C}_{\mathrm{e}} / \mathrm{q}_{\mathrm{e}}$ grafiği çizilmesiyle (Şekil 8) grafiğin eğiminden $\mathrm{q}_{\mathrm{m}}$, ve y ekseninin kesim noktasından $\mathrm{K}_{\mathrm{L}}$ hesaplanmıştır. Langmuir modelinden hesaplanan parametreler Tablo 1'de verilmiştir. Diğer yandan Langmuir modelinin temel karakteristiği, boyutsuz ayırma faktörü $\mathrm{R}_{\mathrm{L}}$ kullanılarak belirlenebilir [21]. $\mathrm{R}_{\mathrm{L}}$ değeri Eş. 4 ile belirlenebilmektedir. $\mathrm{R}_{\mathrm{L}}$ değerinin 0-1 aralığında olması uyumlu adsorpsiyonu, $\mathrm{R}_{\mathrm{L}}>1$ iken adsorpsiyonun uygun olmadığını belirtmektedir [11,12]. Bu çalışmada $\mathrm{R}_{\mathrm{L}}$ değerleri çalışılan boyar madde derişimleri $(25-200 \mathrm{mg} / \mathrm{L})$ için $0,014-0,10$ aralığında hesaplanmıştır. $\mathrm{R}_{\mathrm{L}}$ değerlerine göre AV 90 adsorpsiyonu için PANİ/SA'nın uygun olduğu söylenebilmektedir.

$$
R_{L}=\frac{1}{1+K_{L} C_{O}}
$$

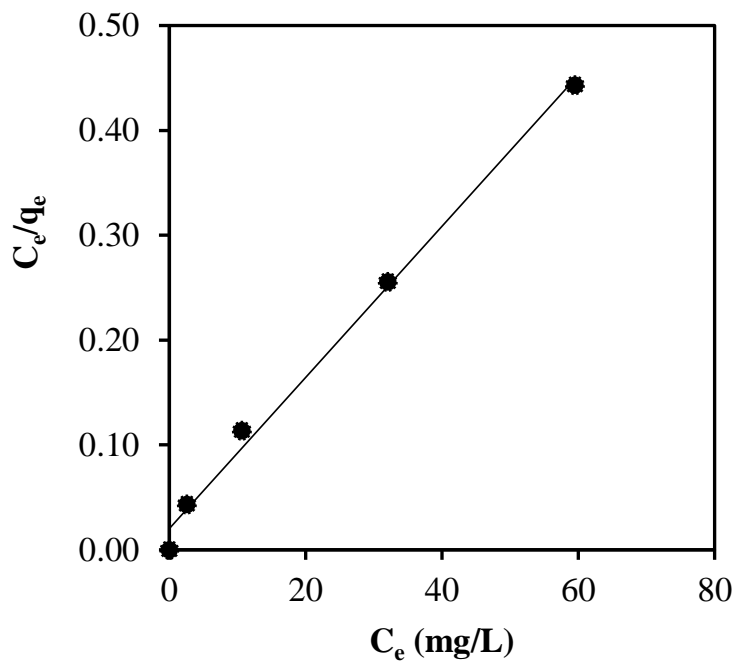

Şekil 8. Langmuir izotermi

Freundlich adsorpsiyon izoterm modeli heterojen yüzeylerde gerçekleşen, tek tabakayla sınırlandırılamayan adsorpsiyonu tanımlamada kullanılmaktadır $[12,20]$. Freundlich izoterm modelini deneysel verilere uyarlamak için eşitliğin doğrusal formu (Eş. 5) kullanılmıştır.

$$
\ln \mathrm{q}_{\mathrm{e}}=\ln \mathrm{K}_{\mathrm{F}}+\frac{1}{n} \ln \mathrm{C}_{\mathrm{e}}
$$

$\mathrm{Bu}$ eşitlikte $\mathrm{K}_{\mathrm{F}}$ ve $\mathrm{n}$, Freundich sabitini ve adsorpsiyon şiddetini ifade etmektedir. 1/n heterojenlik faktörü olup 0-1 arasında değer almakta ve sıfıra yaklaştıkça yüzeyin heterojenlik seviyesi artmaktadır. Freundlich sabitlerini belirlemek için, lnq e'ye karşı $\ln C_{e}$ grafiği çizilerek (Şekil 9) grafiğin eğiminden n sabiti ve y eksenini kesim noktasından $\mathrm{K}_{\mathrm{F}}$ Freundlich sabiti hesaplanmıştır. Freundlich modelinden hesaplanan parametreler Tablo 1'de verilmiştir. 


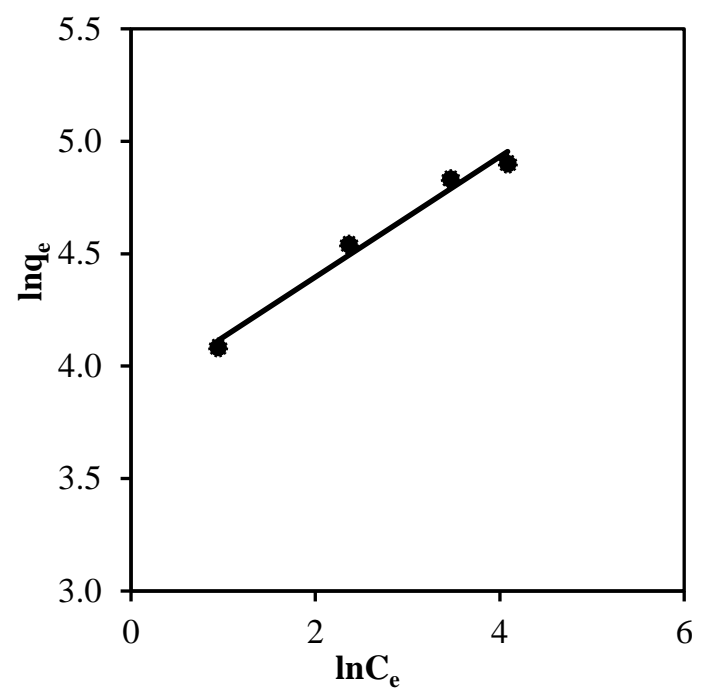

Şekil 9. Freundlich izotermi

Temkin izoterm modelinde adsorbent - adsorbe olan maddeler arasındaki etkileşim göz önüne alınmaktadır. Çözelti içerisindeki tüm moleküllerin adsorpsiyon entalpisi dikkate alınarak geliştirilmiştir [22]. Temkin izoterm eşitliği:

$$
\mathrm{q}_{\mathrm{e}}=\mathrm{q}_{\mathrm{t}} \ln \mathrm{K}_{\mathrm{T}}+\mathrm{q}_{\mathrm{t}} \ln \mathrm{C}_{\mathrm{e}}
$$

Bu eşitlikte, $\mathrm{K}_{\mathrm{T}}$ Temkin izoterm sabiti ( $\left.\mathrm{L} / \mathrm{mg}\right), \mathrm{q}_{\mathrm{T}}=\mathrm{RT} / \mathrm{b}$, ve $\mathrm{b}$ ise adsorpsiyon 1sısı ile ilgili Temkin sabitini $(\mathrm{J} / \mathrm{mol})$ göstermektedir. Temkin izoterm sabitlerini belirleyebilmek için $\ln \mathrm{C}_{\mathrm{e}}$ 'ye karşı $\mathrm{q}_{\mathrm{e}}$ grafiği çizilmiştir (Şekil 10). Temkin modelinden hesaplanan parametreler Tablo 1'de verilmiştir.

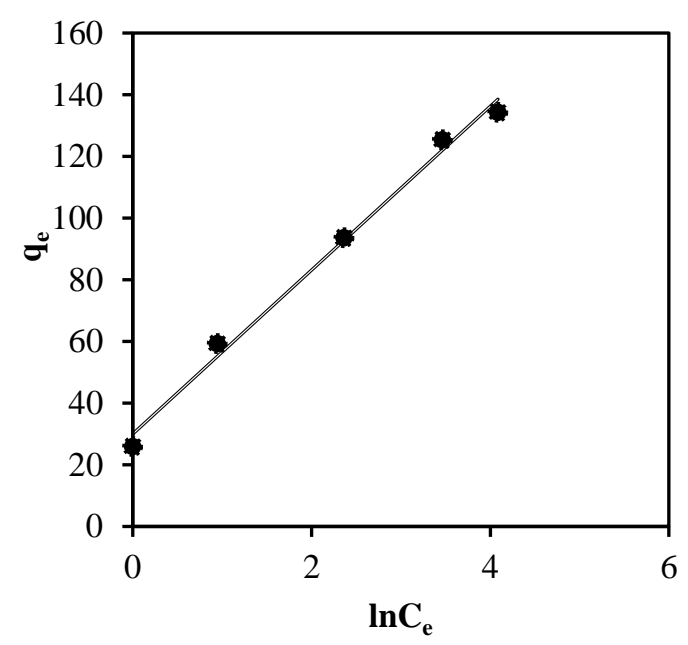

Şekil 10. Temkin izotermi 
Tablo 1. PANI/SA ile AV 90 adsorpsiyon izotermleri

\begin{tabular}{lll}
\hline Adsorpsiyon İzotermleri & Parametreler & Değeri \\
\hline Langmuir & $\mathrm{K}_{\mathrm{L}}$ & $0,35 \mathrm{~L} / \mathrm{mg}$ \\
& $\mathrm{q}_{\mathrm{m}}$ & $138,9 \mathrm{mg} / \mathrm{g}$ \\
& $\mathrm{R}^{2}$ & 0,994 \\
Freundlich & $\mathrm{K}_{\mathrm{F}}$ & 47,6 \\
& $1 / \mathrm{n}$ & 0,27 \\
\multirow{3}{*}{ Temkin } & $\mathrm{R}^{2}$ & 0,981 \\
& $\mathrm{~K}_{\mathrm{T}}$ & $3,1 \mathrm{~L} / \mathrm{mg}$ \\
& $\mathrm{q}_{\mathrm{t}}$ & 26,6 \\
\hline
\end{tabular}

Deneysel sonuçların Langmuir adsorpsiyon izoterm modeline uygulanmasıyla yüksek korelasyon katsayıları $\left(R^{2}=0,994\right)$ elde edilirken, Freundlich $\left(R^{2}=0,981\right)$ ve Temkin modeli $\left(R^{2}=0,992\right)$ ile nispeten daha düşük korelasyon katsayıları elde edilmiştir. Langmuir adsorpsiyon izoterm modelinden elde edilen maksimum adsorpsiyon kapasitesi $\left(\mathrm{q}_{\mathrm{m}}\right)$ 138,9 mg/g olarak bulunmuştur. Ayrıca Freundlich modelinden bulunan $1 / \mathrm{n}$ değeri 0,27 olarak bulunmuş olup adsorbent yüzeyinde heterojen bölgelerin olabileceğini göstermektedir. Her üç modelinde PANi/SA'nın AV 90 adsorpsiyonuna uygun olabileceği söylenebilir. Rafiqi ve diğ., [23] Cu(II)'nin polianilin üzerindeki adsorpsiyon mekanizmasının kompleks olduğunu ve birden fazla mekanizmayla açıklanabileceğini ifade etmektedir. Farklı adsorbentlerle elde edilen adsorpsiyon kapasiteleri Tablo 2'de özetlenmiştir.

Tablo 2. Farklı adsorbentlerle boya gideriminde elde edilen $q_{m}(\mathrm{mg} / \mathrm{g})$ değerleri

\begin{tabular}{|c|c|c|c|}
\hline Adsorbent & Boyar madde & $q_{m}(\mathrm{mg} / \mathrm{g})$ & Kaynak \\
\hline PANİ/Gümüş & Brilliant Green & 49 & [12] \\
\hline Kitosan- PANİ/ZnO & Reaktif Orange 16 & 476,2 & [10] \\
\hline Montmorillonit/Alginat & Basic Red 46 & 35 & [5] \\
\hline Alginat $/ \mathrm{Fe}_{3} \mathrm{O}_{4}$ & Malahit yeşili & 47,84 & [2] \\
\hline PANI/ $/ \mathrm{Bi}_{2} \mathrm{WO}_{6}$ & Congo Red & 141,45 & [3] \\
\hline PANİ & Kristal Violet & 227 & [19] \\
\hline PANI & Metil Orange & 188 & [19] \\
\hline Alginat/Montmorillonit/PANİ & Reaktif Orange 13 & 111,11 & {$[16]$} \\
\hline PANI/SA & Asit Violet 90 & 138,9 & $\mathrm{Bu}$ calısma \\
\hline
\end{tabular}

\subsection{Adsorpsiyon Kinetiği (Adsorption Kinetics)}

AV90 boyar maddesinin PANI/SA malzemesi üzerine adsorpsiyon kinetiği, Lagergren yalancı birinci derece (Eş.7) ve yalancı ikinci derece (Eş.8) kinetik modelleri kullanılarak incelenmiştir.

$$
\begin{aligned}
& \ln \left(q_{e}-q_{t}\right)=\ln \left(q_{e}\right)-k_{1} t \\
& \frac{t}{q_{t}}=\frac{1}{k_{2} q_{e}^{2}}+\frac{t}{q_{e}}
\end{aligned}
$$

Yalancı birinci dereceden kinetiğin deneysel verilere uygulanmasında düşük korelasyon katsayıları elde edilmiş ve bu modelden elde edilen $\mathrm{q}_{\mathrm{e}}$ değerlerinin deneysel $\mathrm{q}_{\mathrm{e}}$ değerleri ile farklılık göstermesinden dolayı boyar madde adsorpsiyon kinetiğinin bu modele uymadığı görülmüştür. Yalanc1 ikinci derece kinetik modelinin ise deneysel verilere uygulanmasıyla (Şekil 11) yüksek korelasyon katsayıları $\left(R^{2}>0,998\right)$ elde edilmiştir (Tablo 3). Yalancı ikinci derece kinetik modelden elde edilen $\mathrm{q}_{\mathrm{e}}$ değerleri ile deneysel $\mathrm{q}_{\mathrm{e}}$ değerlerinin birbirine oldukça yakın olduğu görülmüştür. Yapılan deneyde Asit Violet 90 boyar maddesinin yalancı ikinci dereceden kinetik modeline uyduğu görülmüştür. Lagergren yalancı birinci derece kinetik modelinin deneysel verilere uymamas1, adsorpsiyon prosesinin tamamını tanımlayamamasindan kaynaklanmış olabilir. Yalancı birinci derece kinetik model sadece adsorpsiyon hızının yüksek olduğu 
verilere uygulanabilmektedir. Yalanci ikinci derece kinetik model ise adsorpsiyon prosesinin tümüne uygulanabilir bir modeldir [15]. Ayrıca yalancı ikinci derece kinetik modele göre, adsorpsiyon prosesi adsorbent yüzeyindeki fonksiyonel gruplar ile adsorbat molekülleri arasında kimyasal ve elektrostatik etkileşimlerin olduğu değişik mekanizmaları içermektedir [5]. Bu çalışmada yalancı ikinci derece kinetik model, PANİ/SA yüzeyi ile AV 90 molekülleri arasında elektrostatik etkileşimler olduğunu söyleyebilmektedir. Benzer sonuç PANİ/ferrisiyanid [23], grafen/aljinat [17], PANİ [19] ve PANİ/Bi2 $\mathrm{WO}_{6}$ [3] ile boyar madde adsorpsiyonu çalışmalarında gözlenmiştir.

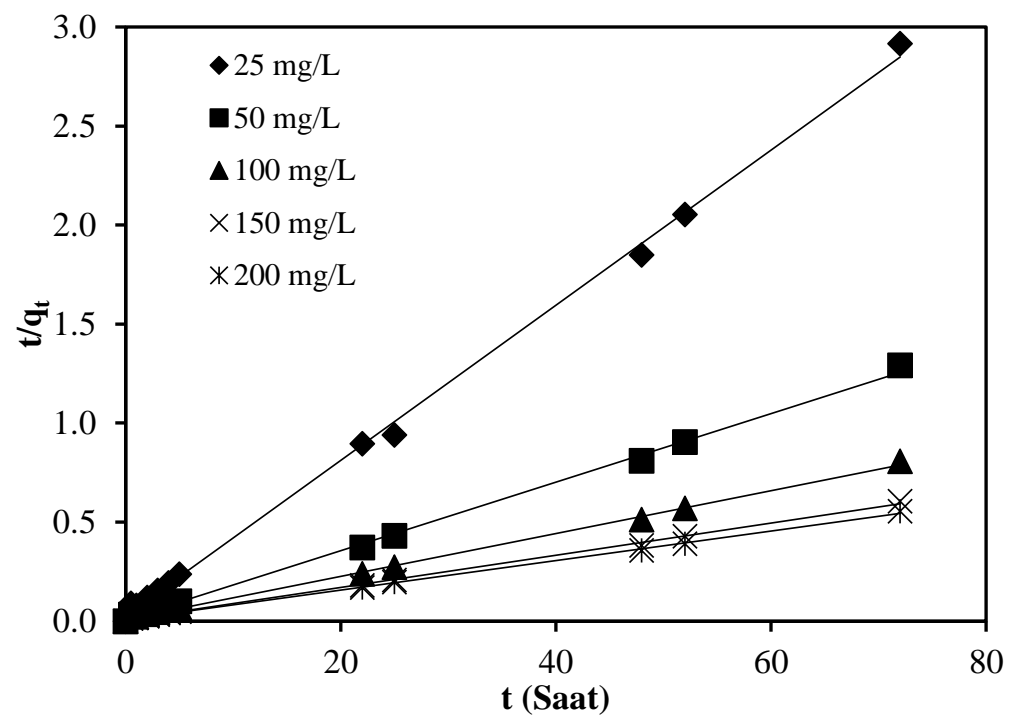

Şekil 11. Yalancı ikinci dereceden kinetik

Tablo 3. Yalancı birinci ve ikinci derece kinetik model sabitleri

\begin{tabular}{ccccccccc}
\hline \multirow{2}{*}{$\begin{array}{c}\mathrm{C}_{\mathrm{o}} \\
(\mathrm{mg} / \mathrm{L})\end{array}$} & $\mathrm{q}_{\mathrm{e}, \text { deney }}$ & \multicolumn{3}{c}{ Yalanc1 birinci derece } & & \multicolumn{3}{c}{ Yalanc1 ikinci derece } \\
\cline { 3 - 4 } \cline { 7 - 9 } & & $\begin{array}{c}\mathrm{q}_{\mathrm{e}, \text { model }} \\
(\mathrm{mg} / \mathrm{g})\end{array}$ & $\begin{array}{c}\mathrm{k}_{1} \\
(1 / \mathrm{saat})\end{array}$ & $\mathrm{R}^{2}$ & & $\begin{array}{c}\mathrm{q}_{\mathrm{e}, \text { model }} \\
(\mathrm{mg} / \mathrm{g})\end{array}$ & $\begin{array}{c}\mathrm{k}_{2} \\
(\mathrm{~g} / \mathrm{mg} . \mathrm{saat})\end{array}$ & $\mathrm{R}^{2}$ \\
\hline 25 & 25,9 & 13,53 & 0,114 & 0,780 & & 25,6 & 0,0530 & 0,999 \\
50 & 59,3 & 3,61 & 0,139 & 0,880 & & 57,8 & 0,0320 & 0,998 \\
100 & 93,7 & 58,02 & 0,12 & 0,954 & & 92,6 & 0,0120 & 0,999 \\
150 & 125,4 & 74,35 & 0,122 & 0,930 & & 123,5 & 0,0085 & 0,999 \\
200 & 134,3 & 76,03 & 0,109 & 0,901 & & 133,3 & 0,0070 & 0,999 \\
\hline
\end{tabular}

\section{SONUÇLAR (CONCLUSIONS)}

Yapılan çalışmada PANİ/SA malzemesi kullanılarak AV 90 boyarmaddesinin adsorpsiyonu incelenmiştir. Ortam başlangıç pH'1, küre sayısı ve boyarmadde derişimi AV 90 adsorpsiyonu üzerinde oldukça önemli parametrelerdir. Yapılan bütün deneylerde adsorpsiyonun dengeye gelme süresi 48 saat olarak belirlenmiştir. Deney sonuçları pH'ın adsorpsiyonda en önemli parametrelerden birisi olduğu ve en yüksek adsorpsiyonun $\mathrm{pH}$ 2'de elde edildiği görülmüsstür. AV 90 anyonik bir boyar maddedir ve pH 2'de elektrostatik kuvvetler daha etkin olmaktadır. Deneylerde 25-200 mg/L boyarmadde derişimi aralığında çalışı $1 \mathrm{~m} ı s ̧ ~ v e ~ 50$ adet küre (kuru ağırlığı $0,06 \mathrm{~g}$ ) ile $25 \mathrm{mg} / \mathrm{L}$ derişimindeki boyarmadde tamamen giderilmiş, $200 \mathrm{mg} / \mathrm{L}$ derişimde olan boyarmadde ise $\% 63$ verimle giderilmiştir. Bu boyarmadde derişimi aralığında 25,9-134,3 mg/g giderim kapasitesi elde edilmiştir. Deneysel verilere Langmuir, Freundlich ve Temkin izoterm modelleri uygulanmış ve her üç modelinde deney verileri ile uygunluk gösterdiği belirlenmiştir. Langmuir modelinden $\mathrm{q}_{\mathrm{m}}$ değeri 138,9 mg/g olarak hesaplanmıştır. AV 90'nın PANİ/SA üzerindeki adsorpsiyon kinetiğinin Yalancı ikinci derece kinetik model ile ifade edildiği görülmüştür. Elde edilen deney sonuçlarına göre PANI/SA adsorbentinin AV 90 boyar maddesinin adsorpsiyonu için oldukça iyi bir alternatif olduğu söylenebilir. 


\section{KAYNAKLAR (REFERENCES)}

[1] Yang, L., Ma, X., Guo, N., "Sodium alginate/Na+-rectorite composite microspheres: preparation, characterization, and dye adsorption", Carbohydrate Polymers, 90, 853-858, 2012.

[2] Mohammadi, A., Daemi, H., Barikani, M., "Fast removal of malachite green dye using novel superparamagnetic sodium alginate-coated $\mathrm{Fe}_{3} \mathrm{O}_{4}$ nanoparticles", International Journal of Biological Macromolecules, 69, 447-455, 2014.

[3] Laabd, M., Ahsaine, H. A., Jaouhari, A., Bakiz, B., Bazzaoui, M., Ezahri, M., Albourine, A., Benlhachemi A., "Congo red removal by $\mathrm{PANi} / \mathrm{Bi}_{2} \mathrm{WO}_{6}$ nanocomposites: Kinetic, equilibrium and thermodynamic studies”, Journal of Environmental Chemical Engineering 4, 3096-3105, 2016.

[4] Patil, M.R., Shrivastava, V.S., "Adsorption removal of carcinogenic acid violet19 dye from aqueous solution by polyaniline- $\mathrm{Fe}_{2} \mathrm{O}_{3}$ magnetic nano-composite”, J.Mater.Environ.Sci. 6 (1), 11-21, 2015.

[5] Hassani, A., Soltani, R. D. C., Karaca, S., Khataee, A., "Preparation of montmorillonite-alginate nanobiocomposite for adsorption of a textile dye in aqueous phase: Isotherm, kinetic and experimental design approaches”, Journal of Industrial and Engineering Chemistry 21, 1197-1207, 2015.

[6] Sarığlu, Ö.F., Keskin, N.O., Çelebioğlu, A., Tekinay, T., Uyar, T., "Bacteria encapsulated electrospun nanofibrous webs for remediation of methylene blue dye in water", Colloids and Surfaces B: Biointerfaces, 152, 245-251, 2017.

[7] Kocaer, F.O., Alkan, U., "Boyar madde içeren tekstil atık sularının arıtım alternatifleri”, Uludağ Üniv. Müh.Mim.Fak.Dergisi, Cilt 7, No 1, 47-55, 2002.

[8] Jain, R., Gupta, V.K., Sikarwa, S., "Adsorption and desorption studies on hazardous dye Naphthol Yellow S", Journal of Hazardous Materials, Cilt 182, 749-756, 2010.

[9] Gengec, E., "Color removal from anaerobic/aerobic treatment effluent of bakery yeast wastewater by polyaniline/beidellite composite materials", Journal of Environmental Chemical Engineering, 3, 24842491, 2015.

[10] Pandiselvi, K., Thambidurai, S., "Synthesis of porous chitosan-polyaniline/ZnO hybrid composite and application for removal of reactive orange 16 dye", Colloids and Surfaces B: Biointerfaces 108, 229238, 2013.

[11] Wang, J., Han, X., Ma, H., Ji, Y., Bi, L., “Adsorptive removal of humic acid from aqueous solution on polyaniline/attapulgite composite”, Chemical Engineering Journal 173, 171- 177, 2011.

[12] Salem, M.A., Elsharkawy, R., Hablas, M.F., "Adsorption of Brilliant Green dye by polyaniline/silver nanocomposite: Kinetic, equilibrium, and thermodynamic studies", European Polymer Journal, 75, 577-590, 2016.

[13] Mu,B., Tang, J., Zhang, L., Wang, A., "Preparation, characterization and application on dye adsorption of a well-defined two-dimensional superparamagnetic clay/polyaniline/Fe3O4 nanocomposite", Applied Clay Science, 132-133, 7-16, 2016.

[14] Olad, A., Naseri, B., "Preparation, characterization and anticorrosive properties of a novel polyaniline/clinoptilolite nanocomposite", Progress in Organic Coatings, 67, 233-238, 2010.

[15] Jiang, N., Xu, Y., Dai, Y., Lou, W., Dai, L., "Polyaniline nanofibers assembled on alginate microsphere for $\mathrm{Cu}^{2+}$ and $\mathrm{Pb}^{2+}$ uptake", Journal of Hazardous Materials,215-216,17-24, 2012.

[16] Ayazi,Z., Khoshhesab, Z.M., Azhar, F.F., Mohajeri, Z., "Modeling and Optimization of Adsorption Removal of Reactive Orange 13 on the Alginate-Montmorillonite-Polyaniline Nanocomposite via Response Surface Methodology”, Journal of the Chinese Chemical Society, 64, 627-639, 2017. 
[17] Zhuang, Y., Yu, F., Chen, J., Ma, J., "Batch and column adsorption of methylene blue by graphene/alginate nanocomposite: Comparison of single-network and double-network hydrogels", Journal of Environmental Chemical Engineering, 4(1), 147-156, 2016.

[18] Karthik, R., Meenakshi, S., "Removal of $\mathrm{Cr}(\mathrm{VI})$ ions by adsorption onto sodiumalginatepolyaniline nanofibers", International Journal of Biological Macromolecules, 72, 711-717, 2015.

[19] Sharma, V., Rekha, P., Mohanty, P., "Nanoporous hypercrosslinked polyaniline: An efficient adsorbent for the adsorptive removal of cationic and anionic dyes", Journal of Molecular Liquids, 222, 1091-1100, 2016.

[20] Lu, T., Xiang, T., Huang, X.L., Li,C., Zhao, W.F., Zhang, Q., Zhao, C.S., "Post-crosslinking towards stimuli-responsive sodium alginate beads fort he removal of dye and heavy metals", Carbohydrate Polymers, 133, 587-595, 2015.

[21] Kaykığlu, G., "Kolemanit ve Üleksit Atı̆̆1 ile Sulu Çözeltilerden Metilen Mavisi Giderimi: Kinetik ve İzoterm Değerlendirmesi”, CBÜ Fen Bil. Dergi., 12(3), 499-509, 2016.

[22] Okur, M., "Tekstil Atık sularındaki Metal Kompleks Boyarmaddelerin Yumurta Kabukları İle Giderimi", Journal of the Faculty of Engineering and Architecture of Gazi University, 28 (4), 777-785, 2013.

[23] Rafiqi, F.A., Majid, K., "Removal of copper from aqueous solution using polyaniline and polyaniline/ferricyanide composite", Journal of Environmental Chemical Engineering, 3, 2492-2501,2015. 